Notre Dame Journal of Formal Logic

Volume 35, Number 2, Spring 1994

\title{
Modal-Epistemic Variants of Shapiro's System of Epistemic Arithmetic
}

\author{
LEON HORSTEN
}

\begin{abstract}
This paper presents formalizations of classical first-order arithmetic which contain a modal and an epistemic operator. The embedding under variants of Gödel's translation of Intuitionistic arithmetic in such systems is discussed, and for one modal-epistemic system of arithmetic a possible worlds semantics is given.
\end{abstract}

1 Introduction This paper discusses modal-epistemic systems of arithmetic. They can be situated among the so-called "epistemic" formulations of arithmetic, of which Shapiro's system of epistemic arithmetic is a paradigmatic example. The next section reviews the basic properties of Shapiro's system. In the third section, it is explained how an analysis of the absolute provability operator of Shapiro's system naturally leads one to develop a modal-epistemic theory of arithmetic. Subsequently a modalepistemic system of arithmetic (MEA) is presented. In the fourth section it is shown that the modal-epistemic system is a conservative extension of Heyting arithmetic. In the fifth section, a possible world semantics for the system is constructed. A soundness theorem is given and a completeness theorem is proved for the logical fragment of the system. In Section six, we exploit the surplus of expressive power of our modal-epistemic analysis of Shapiro's absolute provability operator in constructing a modal-epistemic system of arithmetic on a modal-structural basis. It is suggested that for the modal-structural interpretation of arithmetic to "make sense of" intuitionistic arithmetic, it must assume a stronger non-logical statement than it has hitherto been prepared to do.

2 Shapiro's system of Epistemic Arithmetic (EA) The language of EA ( $\left.L_{\mathrm{EA}}\right)$ contains all the symbols of the formal language of first-order arithmetic $\left(L_{\mathrm{PA}}\right)$, plus an epistemic sentence operator $K$ (which we consider to be a logical symbol). So the only nonlogical symbols are the individual constant $\mathbf{0}$, a one-place function symbol $\mathbf{s}$ (the successor operator), and the two-place function symbols + (plus) and · (times). The 
identity predicate $(=)$ is taken as a logical constant. Terms, formulas and sentences of $L_{\mathrm{EA}}$ are defined in the usual manner. The intended interpretation of $K$ is "is provable in principle."

The theory $E A$ contains the Peano axioms for elementary arithmetic, with its recursive axioms for addition and multiplication. We define $E A$ as the smallest theory which contains these axioms, in which the absolute provability operator $K$ is governed by the (Barcan-free) S4 axioms and rules, and which is closed under (classical) first-order logic.

Let $H A$ stand for the usual formalization of first-order Heyting arithmetic, and let $L_{\mathrm{HA}}$ be the language of this theory. Shapiro then inductively defines a translation $V: H A \rightarrow E A$ (see his [5]). Indicating by means of a subscript $i$ ("intuitionistic") that a formula belongs to $L_{\mathrm{HA}}$, we can paraphrase this definition as follows:

(1) for atomic formulas:

- $V\left(A_{i}\right)=K A$

(2) for complex formulas:

- $V(A \wedge B)_{i}=K\left(V\left(A_{i}\right)\right) \wedge K\left(V\left(B_{i}\right)\right)$

- $V(A \vee B)_{i}=K\left(V\left(A_{i}\right)\right) \vee K\left(V\left(B_{i}\right)\right)$

- $V(A \rightarrow B)_{i}=K\left(K\left(V\left(A_{i}\right)\right) \rightarrow K\left(V\left(B_{i}\right)\right)\right)$

- $V(A \leftrightarrow B)_{i}=K\left(K\left(V\left(A_{i}\right)\right) \leftrightarrow K\left(V\left(B_{i}\right)\right)\right)$

- $V(\neg A)_{i}=K\left(\neg K\left(V(A)_{i}\right)\right)$

- $V(\forall x A(x))_{i}=K\left(\forall x V(A(x))_{i}\right)$

- $V(\exists x A(x))_{i}=\exists x K\left(V(A(x))_{i}\right)$

Beside the "intuitive" plausibility of the definition of Shapiro's translation, the strongest confirmation of the thesis that the meanings of the intuitionistic arithmetical statements can at least in part be expressed in the language of epistemic arithmetic is given by the faithfulness theorem for the translation $V$ (see Goodman [2]):

Theorem 2.1 (Faithfulness theorem for $V$ ) For every sentence $A \in L_{\mathrm{HA}}$ :

$$
\vdash_{\mathrm{HA}} A \leftrightarrow \vdash_{\mathrm{EA}} V(A)
$$

3 A system of modal-epistemic arithmetic (MEA) In EA the sentential operator $K$ is a primitive or unanalyzed operator. But the notion of provability in principle seems to contain both a modal component (provability) and an epistemic component (mathematical proof). Therefore it seems useful to attempt to construct a formal system in which Shapiro's operator $K$ is regarded as a complex operator $\diamond P$, where $\diamond$ is the familiar possibility operator, and the operator $P$ should be read as "some (not further specified) mathematician has a proof that...." We will show that some of the logical properties which Shapiro postulates for the notion of absolute provability (i.e. the axioms for S4) can be derived from more basic logical properties of the notions of possibility and (having a) proof.

The language of $M E A\left(L_{\mathrm{MEA}}\right)$ contains a modal operator $(\diamond)$ and an epistemic operator $(P)$, and the symbols of $L_{\mathrm{PA}}$, except that we will not assume that $L_{\mathrm{MEA}}$ has names for all partial recursive functions (this will simplify our definition of models for $M E A$ in Section 7). 
Terms, formulas and sentences are again defined in the usual way. A formula is called ontic if it contains no occurrences of $\diamond$ or $P$.

We now construct a Hilbert-style deduction system for $M E A$. As our propositional and quantificational axioms and rules we take some standard Hilbert-style formalization of classical predicate logic with identity. The necessity operator of $M E A$ is governed by the $\mathrm{S} 5$ axioms and rules with the Barcan formula, plus the following principle:

M1 $\diamond A \rightarrow A, \quad$ where $\mathrm{A}$ is an ontic sentence.

The intended domain of $M E A$ is the natural number structure. The Barcan formula follows from the idea that the domain does not grow when we pass to accessible possible worlds. Therefore the validity of the Barcan formula follows from the platonistic thesis that the natural numbers structure is the intended domain of all possible worlds. Axiom M1 asserts that all arithmetical truths are necessary. If in this axiom the restriction of the parameter to ontic formulas were omitted, then the modal operator would "collapse." $M E A$ has the following two epistemic axioms:

$$
\begin{array}{ll}
\text { E1 } & P A \rightarrow A . \\
\text { E2 } & P A \rightarrow P P A .
\end{array}
$$

and the following modal-epistemic principles:

$$
\begin{array}{ll}
\text { ME1 } & \text { From } A, \text { infer } \diamond P A . \\
\text { ME2 } & {[\diamond P A \wedge \diamond P(A \rightarrow B)] \rightarrow \triangle P B .}
\end{array}
$$

As arithmetical axioms of $M E A$, we have as before the Peano axioms for elementary arithmetic.

E2 is undoubtedly the most controversial principle of $M E A$. Whether one accepts or rejects it, ultimately depends on one's concept of proof. We regard E2 as plausible, because the mere fact that a mathematician has a proof of a sentence $A$ can be taken to be a proof of the fact that she has a proof of $A$. Note that this is not quite the same as saying that the proof which she has is a proof of the fact that she has a proof of $A$ (proofs of arithmetical sentences are not facts!), although we would also tend to agree with this assertion.

E2 can be objected to from the following standpoint. Suppose one holds that a proof of a sentence $A$ can only be an ordered set of sentences (or propositions) of which $A$ is the last sentence (proposition). Now if one has a proof of $A$, consisting of $n$ sentences (propositions), say, then $A$ (and not $P A$ !) is the last sentence of this sequence. One can add to this proof a line $n+1$, on which one writes $P A$, to which one infers not from a specific set of lines $\left\{n_{1}, \ldots, n_{k}\right\}$, but form the entire proof $\langle 1, \ldots, n\rangle$. But the result will be a proper extension of the original proof. If one takes this position, then the following weakening of $\mathrm{E} 2$ will still be acceptable:

$$
\text { E2* } \quad P A \rightarrow \triangle P P A .
$$

Let us refer to the system which results from replacing E2 in $M E A$ by E2* as $M E A^{*}$. $M E A^{*}$ does not seem to be significantly weaker than $M E A$. Propositions 3.3 and 3.4, as well as the faithfulness theorem 4.1 continue to hold if one replaces $M E A$ by $M E A^{*}$.

Definition 3.1 A formula $A \in L_{\mathrm{MEA}}$ is a $\diamond P$-formula if each subformula of $A$ of the form $P B$ is immediately preceded by an occurrence of $\diamond$. 
Lemma 3.2 For each $\diamond P$-formula $A \in L_{\mathrm{MEA}}, \vdash_{\mathrm{MEA}} \diamond A \rightarrow A$.

Proof: By induction on the complexity of the formula $A$.

The proofs of the following propositions are then straightforward:

Proposition 3.3 For every $\diamond P$-formula $A, \vdash_{\mathrm{MEA}} \diamond P A \rightarrow A$.

Proposition 3.4 $\vdash_{\mathrm{MEA}} \diamond P A \rightarrow \diamond P \diamond P A$.

So we can say that the theory $M E A$ gives a modal-epistemic motivation or justification of two of the four axioms that Shapiro postulates for the notion of absolute provability.

$4 M E A$ is a conservative extension of $H A$ Let $V^{\prime}$ be the translation from $E A$ to $M E A$ which maps every formula $A$ of $L_{\mathrm{EA}}$ to the sentence of $L_{\mathrm{MEA}}$ that results from replacing every occurrence of $K$ in $A$ by an occurrence of $\diamond P$. Now let $V^{*}$ be the following translation. $V^{*}: H A \rightarrow M E A: A \rightarrow V^{\prime}(V(A))$. Then we can prove the following theorem:

Theorem 4.1 (Faithfulness theorem for $V^{*}$ ) For all sentences $A \in L_{H A}$ :

$$
\vdash_{\mathrm{HA}} A \Leftrightarrow \vdash_{\mathrm{MEA}} V^{*}(A) \text {. }
$$

Proof: The left-to-right direction is easy to prove. It follows from ME1, ME2, propositions 3.3 and 3.4, and the soundness of $V$. The right-to-left direction can be proved by means of a variation on Flagg and Friedman's proof of the faithfulness theorem for $V$ (see their [1]).

5 A possible worlds semantics for $M E A$ In his [5], Shapiro emphasizes the difficulty of finding a philosophically interesting semantics for his system $E A$. Here we construct a possible worlds semantics for our system. The models of this semantics can be informally interpreted as follows. Consider an arbitrary mathematician who is occupied solely with proving sentences of $L_{\text {MEA }}$. A possible world of a model can be thought of as a possible situation in which this mathematician has a particular (possibly empty, possibly infinite) collection of statements of $L_{\mathrm{MEA}}$ of which she has a demonstration.

No limitations are imposed on the means which our mathematician has at her disposal for proving such sentences. She may in some possible situation have a higherorder demonstration of the ontic sentence of $L_{\mathrm{MEA}}$ which expresses the consistency of $M E A$. She may in some possible situation "intuitively" see that a statement which is independent of Peano arithmetic expresses an irreducible truth of arithmetic. On the other hand, our mathematician may also in some possible situation fail to draw obvious consequences of statements of which she has a proof. It will be required of all proved sentences in a possible situation that they are true, and a proof of a sentence $A$ will count as a proof of $P A$. Moreover, if $\Delta$ is the collection of sentences of $L_{\text {MEA }}$ which our mathematician has proved in a possible situation $w_{1}$, and $\Theta$ is 
the collection of sentences of $L_{\mathrm{MEA}}$ which she has proved in $w_{2}$, then there must be a possible situation in which she has a demonstration of every sentence of $L_{\mathrm{MEA}}$ which can be derived in $M E A$ from $\triangle \cup \Theta$.

An interpretation of $M E A$ is an ordered triple $\langle W, R, I>$, where $W$ is a set of sets of sentences of $L_{\mathrm{MEA}}, R$ is a relation defined on $W$, and $I$ is a function which assigns denotations to expressions of $L_{\mathrm{MEA}} . I_{[a / x]}$ is the assignment function which is just like $I$ except (possibly) that it assigns the number $a$ to the variable $x$.

$W, R$ and $I$ have to meet the following conditions. Let $N=<\omega, T>$ be the standard interpretation of arithmetic. $N$ is the interpretation of $L_{\mathrm{PA}}$ such that $T$ assigns 0 to $\mathbf{0}$, the successor of $T(\mathbf{t})$ to $s(\mathbf{t})$ for every term $\mathbf{t}$, the addition function to + , and the multiplication function to $\cdot$

For all sets $\Delta, \Theta$ of sentences of $L_{\mathrm{MEA}}$, let $C L(\Delta, \Theta)$ (the closure of $\Delta$ and $\Theta$ ) be the set of sentences derivable in MEA from $\triangle \cup \Theta$.

$W$ is a set of sets of sentences of $L_{\mathrm{MEA}}$ such that:

(1) $\forall w \in W, \forall A \in L_{\mathrm{MEA}}$, if $A \in w$, then $P A \in w$.

(2) $\forall w \in W, \forall A \in L_{\mathrm{MEA}}$, if $A \in w$, then $I(A, w)=\mathrm{l}$.

Informally, we can refer to the elements of $W$ as the possible worlds of the interpretation $\langle W, R, I\rangle . \quad R$ is a reflexive, symmetrical and transitive relation on $W$ such that $\forall w_{h}, w_{i}, w_{j}$, if $w_{h} R w_{i}$ and $w_{h} R w_{j}$; then there is a $w_{k} \in W$ such that $C L\left(w_{i}, w_{j}\right)<w_{k}$ and $w_{h} R w_{k}$. Informally, we may think of $R$ as the accessibility relation defined on $W$. The assignment function $I$ satisfies the following conditions:

(1) For all terms $t$, for all relation symbols $R$, for all $w \in W: I(w, t)=T(t) ; I(w, R)=T(\mathbf{R})$; for all atomic sentences $\mathbf{R}\left(\mathbf{t}_{1}, \ldots, \mathbf{t}_{n}\right)$, and for all $w \in W: I\left(w, \mathbf{R}\left(\mathbf{t}_{1}, \ldots, \mathbf{t}_{n}\right)\right)=I(w, \mathbf{R})$ $<I\left(w, \mathbf{t}_{1}\right), \ldots, I\left(w, \mathbf{t}_{n}\right)>$

(2) If $A=B \rightarrow C$, then for all $w \in W: I(w, A)=1$ if $I(w, B)=0$ or $I(w, C)=1$; otherwise $I(w, A)=0$;

(3) If $A=\neg B$, then for all $w \in W: I(w, A)=1$ if $I(w, B)=0$; otherwise $I(w, A)=0$;

(4) If $A=\exists x B(x)$, then for all $w \in W: I(w, A)=1$ if $I_{[a / x]}(B(x))=1$ for some $a \in \omega ; I(w, A)=0$ otherwise;

(5) If $A=\diamond B$, then for all $w \in W: I(w, A)=1$ if for some $w^{\prime} \in W$ such that $w R w^{\prime}, I\left(w^{\prime}, B\right)=1$; $I(w, A)=0$ otherwise;

(6) If $A=P B$, then for all $w \in W: I(w, A)=1$ if $B \in w ; I(w, A)=0$ otherwise.

The notions of logical truth, truth under an interpretation (at a possible world), logical consequence are then introduced in the usual way.

It is routine to show that this semantics is sound for $M E A$. Since $M E A$ contains Peano arithmetic, we cannot prove a completeness theorem for our semantics. However, using the method of canonical models (as described in Hughes and Cresswell [4]), we can prove in a fairly straightforward way a completeness theorem for the logical fragment of $M E A$, i.e. the theory which results from removing the Peano axioms from $M E A$. Of course we then have to generalize our definition of interpretation in the obvious way to allow different domains (not only the standard model of arithmetic) and different interpretations of the nonlogical symbols. 


\section{Application: Epistemic Arithmetic in a modal-structural setting}

6.1 The modal-structural interpretation and constructivistic arithmetic Here the modal-structural interpretation of arithmetic is a formalized philosophical interpretation of classical arithmetic which tries to escape mathematical platonism (see Hellman [3]). It claims that the logical structure of an arithmetical sentence differs from its surface structure, and that this logical structure contains a modal component. Where classical arithmetic seems to posit the actual existence of an infinite structure of abstract objects, the modal structuralist claims that all that it really posits is the possible existence of a structure isomorphic with this platonistic structure.

Any philosophical interpretation of arithmetic must meet the requirement of explaining the intelligibility of constructivistic arithmetic even for the classical mathematician, and the fact that constructivistic arithmetic forms indeed a part of arithmetic. Shapiro's translation $V$ (see Section 2) can be seen as an attempt by the platonistic interpretation to meet this requirement: it can be argued that classical mathematicians understand intuitionistic arithmetic under something like this translation. If the modal-structural interpretation wants to satisfy this requirement, it must show that it can also interpret constructivistic arithmetic under something like Shapiro's translation. We will investigate to what extent this is possible.

In order to define such a variant of the translation $V$, an epistemic formalization of the modal-structural interpretation must be constructed. The language of the formalized modal-structural interpretation contains a modal operator. We have argued in this paper that a modal operator is also "hidden" in the absolute provability operator of $L_{\mathrm{EA}}$, and we have tried to explicate this operator in $L_{\mathrm{MEA}}$. In the formalization of a modal-structural interpretation, then, we seem to really need the resulting stronger expressive power of $L_{\mathrm{MEA}}$. Otherwise we risk suppressing logical properties of the modal and the epistemic operator and logical relations between them, which seem to be especially important in a modal-structural setting.

6.2 A modal-structural system of Epistemic Arithmetic (MSEA) For the sake of simplicity, we base the system which we are going to construct on Primitive Recursive Arithmetic (PRA), as described in Smorynski [6]. Let $P R A_{f}$ be a onesentence formulation of $P R A$. We have seen that the modal-structural interpretation only assumes the possible existence of the natural numbers. $V *$ translates existential formulas of $L_{\mathrm{HA}}$ into existential formulas of $L_{\mathrm{MEA}}$, which posit the existence of numbers. Therefore it seems natural in a modal-structural context to slightly modify the translation function $V^{*}$. We replace $V^{*}$ by the translation function which translates every sentence $A \in L_{\mathrm{HA}}$ into $\diamond V^{*}(A)$, and refer to this translation as $V^{m s}$. The aim of course is to faithfully interpret intuitionistic Primitive Recursive Arithmetic (HPRA) under $V^{m s}$.

The most straightforward approach to adapting the framework of $M E A$ to a modal-structural setting is to drop M1 and the Barcan formula from $M E A$ and to replace the Peano axioms by $\diamond\left(P R A_{f}\right)$. Unfortunately the resulting system is too weak to interpret HPRA under $V^{m s}$. Roughly and intuitively, what we need is that at some possible world, $P R A_{f}$, as well as ME1 and ME2, propositions 3.3 and 3.4 hold, which is more than this modal-structural system can guarantee. Somehow it needs to be strengthened.

One way in which this can be done is to strengthen $\diamond\left(P R A_{f}\right)$ to $\diamond P\left(P R A_{f}\right)$, and to make sure that ME1, ME2, $\diamond P(\diamond P A \rightarrow A)$ for every $\diamond P$-formula $A$, and proposition 
3.4 hold. (Probably some of these could, as in the case of $M E A$, be derived from more basic modal and epistemic principles). Yet since $\diamond P\left(P R A_{f}\right)$ says that there might have been a situation in which some mathematician had a proof that model of PRA actually exists in that situation, this solution involves a strong idealization of the epistemic powers of actual mathematicians.

For this reason we propose an alternative system, which we call MSEA. The language of $M S E A$ is the same as the language of $M E A$. Its modal operator is governed by the 44 principles. MSEA does not contain the "platonistic" principles M1 and the Barcan formula, but it does contain all epistemic and modal-epistemic principles which $M E A$ contains (E1, E2, ME1 and ME2). As the non-logical axiom of MSEA we take:

MPRA $_{f}: \quad \diamond \square\left(P R A_{f}\right)$

This axiom says (roughly) that the natural number structure might have necessarily existed. This concludes the description of MSEA.

MPRA $_{f}$ is stronger than the kind of assumption that modal structuralists typically posit $\left(\diamond\left(P R A_{f}\right)\right)$. Perhaps this is the price the modal structuralist has to pay for explicating the intelligibility of constructivistic mathematics for the classical mathematician. But $M S E A$ does not carry with it the strong idealization that $\diamond P\left(P R A_{f}\right)$ implies.

MSEA does presuppose that the statement that there might have been a structure which, if it existed, would necessarily exist does not imply that such a structure necessarily (and hence actually) exists. In other words, $M S E A$ finds the characteristic axiom of S5 (which is by most defenders of the modal-structural interpretation taken to be acceptable) too strong. It is not hard to see that we have:

Theorem 6.1 (Faithfulness of $V^{m s}$ ) For all $A \in L_{\mathrm{HA}}: \vdash_{\mathrm{HPRA}} A \Leftrightarrow \vdash_{\mathrm{MSEA}}$ $V^{m s}(A)$.

So we have an epistemic formulation of the modal-structural interpretation based on a relatively meager part of arithmetic, which can account for an equally meager part of intuitionistic arithmetic under something like Shapiro's translation. It seems that analogous faithfulness results can be proved for epistemic formalizations of the modal-structural interpretation which are constructed along the lines of MSEA except for being based on a stronger arithmetical theory (e.g. on second-order Peano arithmetic).

Acknowledgment I want to thank Tony Anderson for extremely valuable comments on earlier versions of this paper. The research for this paper was sponsored by the Belgian National Fund for Scientific Research, which is gratefully acknowledged.

\section{REFERENCES}

[1] Friedman, H., and R. C. Flagg, "Epistemic and intuitionistic formal systems," Annals of Pure and Applied Logic, 32 (1986), pp. 53-60.

[2] Goodman, N., "Epistemic arithmetic is a conservative extension of intuitionistic arithmetic," Journal of Symbolic Logic, 49 (1984), pp. 192-203. 
[3] Hellman, G., Mathematics Without Numbers. Towards a Modal-Structural Interpretation, Clarendon Press, Oxford, 1989.

[4] Hughes, G., and M. Cresswell, A Companion to Modal Logic, Methuen, New York, 1984.

[5] Shapiro, S., "Epistemic and Intuitionistic Arithmetic," pp. 1-46 in Intensional Mathematics, edited by S. Shapiro, North-Holland, Amsterdam, 1985.

[6] Smorynski, C., Self-reference and Modal Logic, Springer Verlag, New York, 1985.

Center for Logic and Philosophy of Science

Institute for Philosophy

University of Leuven

Kardinaal Mercierplein 2, Leuven

B-3000, Belgium

e-mail: Leon.Horsten@arts.kuleuven.ac.be 